\title{
Fuzzy Logic Approach to Corporate Strategy Mapping
}

\author{
Nooraini Yusoff, Fadzilah Siraj and Siti Maimon Kamso \\ Faculty of IT \\ Universiti Utara Malaysia, 06010 Sintok \\ Kedah, Malaysia \\ Tel: +604-9284629, Fax: +604-9284753, E-mail: nooraini@uum.edu.my, fad173@uum.edu.my
}

\begin{abstract}
Corporate strategy mapping involves an analysis of company's present situation based on strategic factors known as SWOT factors that represent Strengths, Weaknesses, Opportunities, and Threats. The company survival analysis aims to forecast appropriate strategies to undertake. For this purpose, Internal-External Matrix (I-E Matrix) is used to map a company's external and internal factors' scores to determine the overall corporate strategy of a company. Based on both scores, IE matrix recommends a company with three types of strategy; Grow and Build, Hold and Maintain, and Harvest and Divest. In allocating the strategies, there are regions whereby the coordinates of mapped IFA and EFA scores are not able to immediately indicate the appropriate strategy to be undertaken by a company. When such cases arise, an analyst opinion is required in order to determine which strategy implementation is most appropriate. Different analyst may provide different opinion based on his or her assumption, 'market driven' or 'resource-based'. There is no exact solution for the scores that fall in the ambiguous regions. As a solution, one possible approach is to integrate Fuzzy Logic technique with I-E Matrix in producing the automatic strategy formulation. This is due to the fact that Fuzzy Logic has shown to have ability to improve the intelligence of systems on uncertain, imprecise and noisy environment. In this study, Fuzzy Logic has been developed and tested on real cases data. The result shows that the proposed technique is able to forecast the strategic choice for the ambiguous locations that exists in the company.
\end{abstract}

Keywords:

Fuzzy Logic, Corporate Strategy, InternalExternal Matrix, Ambiguous regions,

\section{INTRODUCTION}

Corporate strategy mapping involves the management of a company scans factors that are most important to the corporation's future and forecasts appropriate strategies based on the current situation of the company. The factors are referred to as strategic factors and summarized with the acronym S.W.O.T., standing for Strengths, Weaknesses, Opportunities, and Threats [9]. When performing a strategy decision making, management allocates strategies based upon the interaction of the strategic factors, which have been identified [7] [6].

In running such company's survival analysis, Internal-External (I-E) Matrix is a widely used tool to allocate the SWOT factors and to forecast the strategy based on the company's present situation.

The corporate strategy mapping using I-E Matrix as proposed by [2], is initiated by the External Factor Analysis $(E F A)$ as well as the Internal Factor Analysis (IFA). Both IFA and $E F A$ scores are computed and the values are mapped onto the matrix to forecast the overall corporate strategy of the organization (see Fig. $1)$.

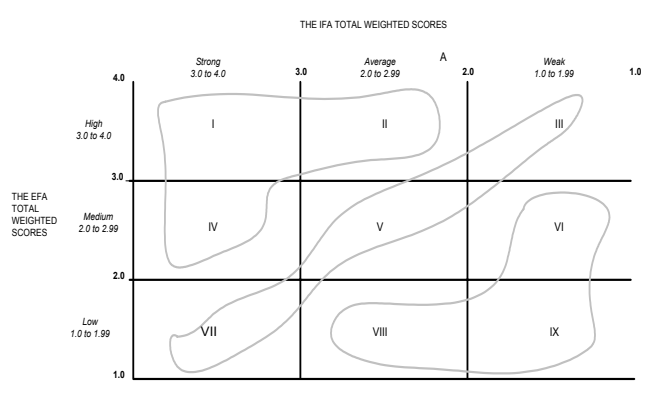

Fig. 1. Internal-External Matrix by David (1999).

The IE Matrix is based on two key dimensions: the IFA total weighted scores on $x$-axis and the $E F A$ total weighted scores on the $y$-axis. On the $x$-axis of IE Matrix, an IFA total weight score of 1.0 to 1.99 represents a weak internal position, a score of 2.0 to 2.99 is considered average, and a score of 3.0 to 4.0 is strong. Similarly, on the $y$-axis, an EFA total weight score of 1.0 to 1.99 is considered low, a score of 2.0 to 2.99 is medium, and a score of 3.0 to 4.0 is high. The IE Matrix can be divided into three major regions that have different strategy implementations. The prescription for divisions that fall into cells I, II or IV can be described as Grow and Build. Divisions that fall into the cells III, V or VII can best be managed with Hold and Maintain strategies. Common prescription for divisions that fall into cells VI, VIII or IX is Harvest and Divest. 


\section{AMBIGUOUS STRATEGY MAPPING}

In running analysis of a company, the decision-making activity involves decisions that are usually made based on uncertain, incomplete, probabilistic, vague knowledge and the outcomes that do not always turn out to be best in hindsight [1]. For the problem in hand, by using the I-E Matrix to map strategy, there are ambiguous regions whereby the coordinates are not able to immediately indicate the appropriate strategies to be undertaken.

For discussion purposes, this paper highlighted four ambiguous regions. The first is a point on the margin shown as ' $\mathbf{A}$ ' in the Fig. 2. The second ambiguity is at the extremities of a cell e.g. between ' $\mathbf{B}_{\mathbf{1}}$ ', ' $\mathbf{B}_{\mathbf{2}}$ ' and ' $\mathbf{B}$ '. The third ambiguity is at the intersections i.e. external to a cell as shown in ' $\mathbf{C}_{\mathbf{1}}$ ', and ' $\mathbf{C}_{\mathbf{2}}$ ' ' and fourthly at equal accumulated values of the two sets of coordinates e.g. at ' $\mathbf{D}_{1}$ ', $(I F A=2.5$ and $E F A$ $3.5)$ and at ' $\mathbf{D}_{2}$ ', $(I F A=2.2$ and $E F A=3.8)$.

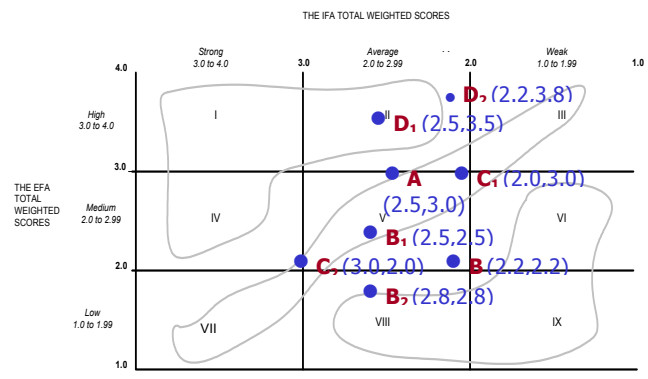

Fig. 2. I-E Matrix with ambiguous strategy mapping

The situation may require the expert opinion in the area to determine which strategy implementation is best to choose. Different analyst may provide different opinion based on his or her assumption, 'market driven' or 'resource-based'. For clarities, consider a company situation mapped by A, 'market driven' analyst would suggest the company to grow and build, meanwhile a 'resource-based' would recommend the harvest and divest strategies. There is no exact solution for the scores that fall in the ambiguous regions. Nevertheless, if there is additional information on the degree of belief to undertake certain strategy, this will give more precise estimation to a company in forecasting the strategy and plan appropriate actions within certain period of time.

Hence this study attempts to explore the potential use of Fuzzy Logic approach in forecasting the corporate strategy. In this study, both IFA and EFA scores mapped onto the IE Matrix are attached a certainty value, $\mu$. This certainty value explains the degree of belief for implementing particular predicted strategy. Thus, an example of fuzzy rule can be written as,

IF IFA score = "weak" $(\mu=0.3)$ AND EFA score $=$ "medium" $(\mu=0.1)$

THEN Harvest and Divest $(\mu=0.65)$ OR Hold and Maintain $(\mu=0.35)$.

With the conventional I-E matrix interpretation, a company with such situation would plan for harvest and divest, but even the confidence value also indicates the same strategy, with appropriate actions to hold and maintain the company still have a chance to improve.

\section{FUZZY LOGIC APPROACH}

Fuzzy systems have attracted the growing interests of researchers in various fields. The number and variety of applications of Fuzzy Logic have been increasing, ranging from consumer products to information systems and decision analysis. Fuzzy Logic has shown to have ability to improve the intelligence of systems on uncertain, imprecise and noisy environment [10] [4] [3].

In this study, Fuzzy Logic has been applied to forecast a feasible strategy for a particular organization. The steps involved as proposed by [5] are depicted in Fig 3.

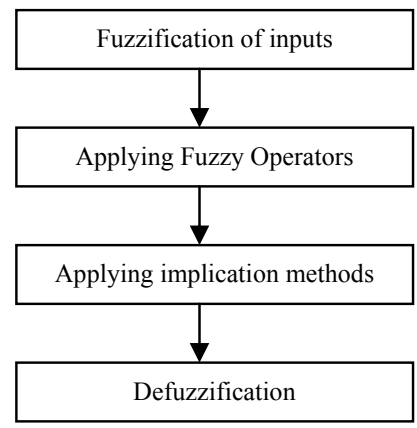

Fig. 3. Steps in Fuzzy Logic

\section{A. Fuzzification of Inputs}

Fuzzification is the process of transformation of crisp values to the corresponding values in fuzzy values [5]. For this purpose, the fuzzifier takes the inputs and determines the degree to which they belong to each of the 
appropriate fuzzy sets via membership functions.

In this study, two variables have been identified as input to Fuzzy Logic System, namely the EFA and IFA scores. The output variable is represented by the Strategy feasibility. For the input variables, a lambda membership function was used to define the labels for the fuzzy variables. Thus the input variables can be defined in the following way:

\section{$E F A \in\{l o w$, medium, high\} \\ IFA $\in\{$ weak, average, strong\}}

For illustration purposes, the membership graph for IFA and EFA are shown in Fig. 4 and Fig 5 respectively.

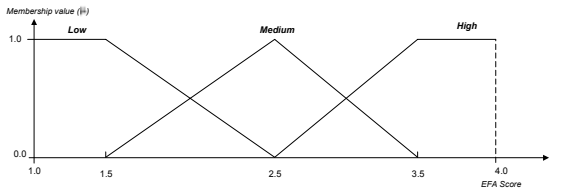

Fig. 4. Membership function graph of $E F A$ Score

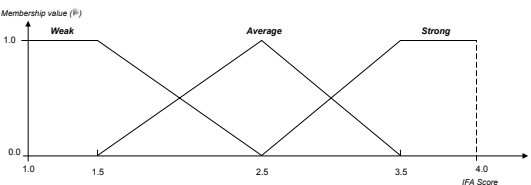

Fig. 5. Membership function graph of IFA Score

For the output variable a lambda membership function is also used to a define Strategy feasibility such that,

Strategy feasibility $\in\{$ Harvest and Divest, Hold and Maintain, Grow and Build\}

\section{B. Applying Fuzzy Operators}

Fuzzy rules combine two or more input fuzzy sets, called the antecedent sets, and associate with them an output, or consequent set. The antecedent sets are combined by means of operators that are analogous to the usual logical conjunctives "and" or "or" [5] [3]. An example of a fuzzy rule for forecasting strategy problem might be:

IF the IFA score = "Weak" AND EFA score = "Low", THEN implement the Harvest and Divest strategy.

One method of storing and representing fuzzy rules is through the use of a fuzzy associate memory (FAM) matrix [8]. Fig. 6 shows an example of a FAM matrix. As indicated earlier, IFA score input variable has three fuzzy sets associated with it: weak, average and strong. Consequently, EFA score input variable has three associated fuzzy sets: low, medium and high. The output variable, Strategy Feasibility also used lambda membership graph, it also has three fuzzy sets associated with it: Harvest and Divest, Hold and Maintain, and Grow and Build.

\begin{tabular}{|c|c|c|c|}
\hline Low & Weak & Average & Strong \\
\hline Medium & $\begin{array}{c}\text { Harvest and } \\
\text { divest }(H D) \\
\mu_{0}\end{array}$ & $\begin{array}{c}\text { Harvest and } \\
\text { divest }(H D) \\
\mu_{1}\end{array}$ & $\begin{array}{c}\text { Hold and } \\
\text { maintain }(H M) \\
\mu_{2}\end{array}$ \\
\hline High & $\begin{array}{c}\text { Harvest and } \\
\text { divest }(H D) \\
\mu_{3}\end{array}$ & $\begin{array}{c}\text { Hold and } \\
\text { maintain }(H M) \\
\mu_{4}\end{array}$ & $\begin{array}{c}\text { Grow and } \\
\text { build }(G B) \\
\mu_{5}\end{array}$ \\
\hline $\begin{array}{c}\text { Hold and } \\
\text { maintain }(H M) \\
\mu_{6}\end{array}$ & $\begin{array}{c}\text { Grow and } \\
\text { build }(G B) \\
\mu_{7}\end{array}$ & $\begin{array}{c}\text { Grow and } \\
\text { build }(G B) \\
\mu_{8}\end{array}$ \\
\hline
\end{tabular}

Fig. 6. FAM Matrix

\section{Applying implication methods}

In this study, the MIN implication method has been used to obtain a single value given by the antecedent. For example, from Fig. 6, there are 4 cells with non-zero confidence values. Therefore, the single value for each rule invoked is calculated as follow:

$$
\begin{aligned}
\mu_{1} & =\min \left\{I F A_{\text {Average }}(2.5), E F A_{\text {Low }}(3.0)\right\} \\
& =\min \{1.0,0.5\} \\
\mu_{2} & =\min \left\{I F A_{\text {Strong }}(2.5), E F A_{\text {Low }}(3.0)\right\} \\
& =\min \{1.0,0.5\} \\
\mu_{4} & =\min \left\{I F A_{\text {Average }}(2.5), E F A_{\text {Medium }}(3.0)\right\} \\
& =\min \{1.0,0.5\} \\
\mu_{5} & =\min \left\{I F A_{\text {Strong }}(2.5), E F A_{\text {Medium }}(3.0)\right\} \\
& =\min \{1.0,0.5\}
\end{aligned}
$$

\section{Defuzzification}

Once the consequent is evaluated, the defuzzification phase is performed to transform the fuzzy values to crisp values. To illustrate the calculation involves in the defuzzification phase, assume that the shaded cells in Fig. 6 have non-zero confidence values.

The defuzzification is calculated as follow :

Strategy feasibility $=\underline{\mu}_{1} \underline{\mathrm{HD}}+\underline{\mu}_{2} \underline{\mathrm{HM}}+\underline{\mu}_{4} \underline{\mathrm{HM}}+\underline{\mu}_{\underline{5}} \underline{\mathrm{GB}}$ $\mu_{1}+\mu_{2}+\mu_{4}+\mu_{5}$

The actual values of $H D, H M$ and $G B$ are calculated from the membership function graph of Strategy feasibility. For example, if 
the confidence value, $\mu_{1}=0.4$ and the actual value of HD is read from the following graph (Fig. 7).

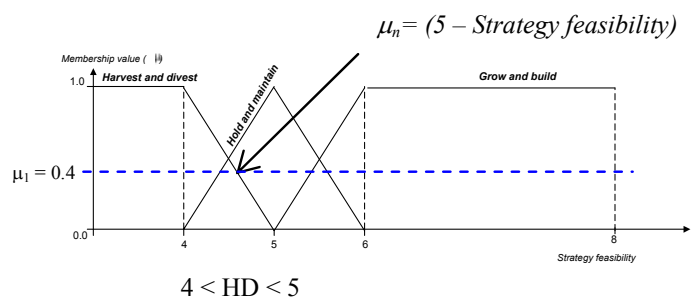

Fig. 7. Membership function graph of Strategy feasibility

Hence strategy feasibility for HD is approximately 4.6. The values of HM and GB could be obtained in a similar way.

\section{CASE STUDY}

The proposed model was first tested using simulated data. Both distinct extreme cases were applied to the model in order to demonstrate how the model performed the prediction. This study highlighted 4 ambiguous regions (denoted as $A, B, C$ and $D$ ) as depicted in Fig. 2. For the purpose for determining the strategy for the coordinate of IE Matrix that fall into the ambiguous regions, for brevity, consider the following cases:

\section{A. Ambiguous Region I}

Coordinate $\boldsymbol{A}, \quad I F A=2.5 E F A=3.0$

From Fig. 2 both values are mapped onto the IE Matrix, and then the region that the values fall into indicates the Average internal factor and High external factor. This condition will suggest that company can Grow and Build based on the rule:

IF IFA is Average AND EFA is High, THEN Grow and Build

Using Fuzzy Logic approach, the IFA and $E F A$ scores are entered into the system as the crisp values.

$$
\left.\begin{array}{l}
I F A=2.5 \\
E F A=3.0
\end{array}\right\} \text { Crisp values }
$$

The confidence value for $I F A$ score $=2.5$ is read from the $I F A$ membership function graph (Fig. 8).

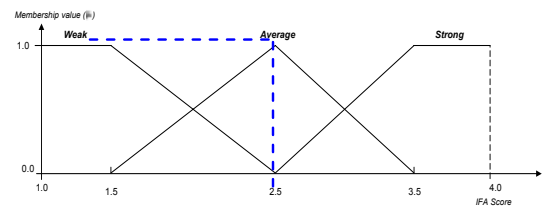

Fig. 8. Membership function graph of IFA Score

Hence from the graph, the value of $\mu$ is 1.0 , and the label for IFA is average.

Thus, $I F A=2.5$

Fuzzy Logic Value (FLV) $\in\{$ Weak (0), Average (1), Strong (0)\}

Similarly, the $E F A$ value of 3.0 has confidence value $(\mu)$ of 0.5 and the label is average or high (see Fig. 9).

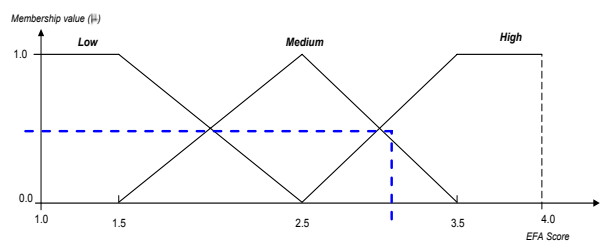

Fig. 9. Membership function graph of EFA Score

$E F A=3.0$

FLV $\in\{$ Low (0), Medium (0.5), High (0.5) $\}$

When both IFA and EFA confidence values are obtained, the FAM matrix is computed to invoke the relevant rules (indicated by the shaded cells).

\begin{tabular}{|c|c|c|c|}
\hline Low & Weak & Average & Strong \\
\hline Medium & $\begin{array}{c}\text { Harvest and } \\
\text { divest }(H D) \\
\mu_{0}\end{array}$ & $\begin{array}{c}\text { Harvest and } \\
\text { divest }(H D) \\
\mu_{1}\end{array}$ & $\begin{array}{c}\text { Hold and } \\
\text { maintain }(H M) \\
\mu_{2}\end{array}$ \\
\hline High & $\begin{array}{c}\text { Harvest and } \\
\text { divest }(H D) \\
\mu_{3}\end{array}$ & $\begin{array}{c}\text { Hold and } \\
\text { maintain }(H M) \\
\mu_{4}\end{array}$ & $\begin{array}{c}\text { Grow and } \\
\text { build }(G B) \\
\mu_{5}\end{array}$ \\
\hline $\begin{array}{c}\text { Hold and } \\
\text { maintain }(H M) \\
\mu_{6}\end{array}$ & $\begin{array}{c}\text { Grow and } \\
\text { build }(G B) \\
\mu_{7}\end{array}$ & $\begin{array}{c}\text { Grow and } \\
\text { build }(G B) \\
\mu_{8}\end{array}$ \\
\hline
\end{tabular}

Fig. 10. FAM Matrix

From Fig. 10, only label Average has non-zero confidence value. Fig. 10 also indicates that label Medium and High have non-zero confidence value. Therefore the FAM cell that would be invoked is shaded in Fig. 10.

$$
\begin{aligned}
\mu_{4} & =\min \left\{I F A_{\text {Average }}(2.5), E F A_{\text {Medium }}(3.0)\right\} \\
& =\min \{1.0,0.5\} \\
\mu_{7} & =\min \left\{I F A_{\text {Average }}(2.5), E F A_{\text {High }}(3.0)\right\} \\
& =\min \{1.0,0.5\}
\end{aligned}
$$


From Fig. 11, when $\mu=0.5$ then, the strategy feasibility for HM and GB equal to 5.5.

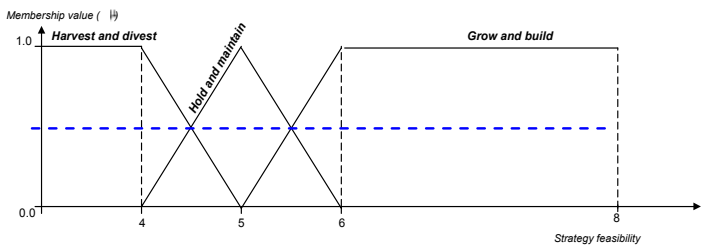

Fig. 11. Membership function graph of Strategy feasibility

$$
\text { Strategy feasibility }=\underline{\mu}_{\underline{4}} \frac{\mathrm{HM}+\underline{\mu}_{7}}{\mu_{4}+\mu_{7}} \frac{\mathrm{GB}}{{ }_{7}}
$$

Strategy feasibility $\in\{\mathrm{HD}(0), \mathrm{HM}(0.5), \mathrm{GB}$ $(0.5)\}$

When the rule:

IF $I F A=2.5$ AND $E F A=3.0$

THEN the resulting consequent of Hold and Maintain yields confidence value of 0.5.

OR Grow and Build yields confidence value of 0.5 .

Similarly, the same approach is applied for the other defined ambiguous regions. The NonFuzzy decision is compared to the Fuzzy decision derived. The results are depicted in Table 1.

To test the validity of the proposed model real cases data were used. The findings from the simulated and real cases indicate that Fuzzy Logic technique can produce satisfactory results when compared with the traditional method. In addition, the findings also reveal that Fuzzy Logic can handle ambiguous situation. The results for ambiguous situations have been verified by the expert. Hence the prototype developed in this study has successfully predicted the ambiguous and nonambiguous cases.

In dealing with each of the position in the ambiguous regions, a basic assumption is necessary as a guide. A strategic management decision maker will have to assume the factors of priority, which influence the decisionmaking. It is either one that is 'market-driven' or 'resource-based'. The 'market-driven' assumption relies a great deal on the external influences of opportunity and threat to affect a strategy, whereas, the resource-based-driven assumption relies on the strengths of resources necessary to undertake a strategy.

Given the above assumption at the ambiguous region of ' $\mathrm{A}$ ' $(I F A=2.5, E F A=3.0)$ if one is 'market-driven', the strategy necessary would be 'Build and Grow' rather than 'Hold and Maintain'. The confidence values are (HD (0), HM (0.5), GB (0.5)).

In the case of region II, i.e. within a cell, 'B' would treated as 'Harvest and Divest' $(I F A=2.2, E F A=2.2),(\mathrm{HD}(1), \mathrm{HM}(0), \mathrm{GB}(0))$; $\mathrm{B}_{1}$ would be treated as a certain 'Hold and Maintain' position (IFA=2.5, $E F A=2.5)$, $(\mathrm{HD}(0), \mathrm{HM}(1), \mathrm{GB}(0))$; and finally $\mathrm{B}_{2}$ $(I F A=2.8, E F A=2.8)$ would be treated as marginal 'Hold and Maintain' position $((\operatorname{HD}(0), \operatorname{HM}(0.525), \mathrm{GB}(0.475))$.

TABLE I

\begin{tabular}{|c|c|c|c|c|c|}
\hline \multirow{2}{*}{$\begin{array}{c}\text { Ambiguous } \\
\text { Region }\end{array}$} & \multirow{2}{*}{ Coordinate } & \multirow{2}{*}{ IFA } & \multirow{2}{*}{ EFA } & \multicolumn{2}{|c|}{ Strategy Feasibility } \\
\hline & & & & Non-Fuzzy & Fuzzy \\
\hline \multirow{3}{*}{ II } & B & 2.2 & 2.2 & Hold and Maintain (HM) & $\{\mathrm{HD}(1), \mathrm{HM}(0), \mathrm{GB}(0)\}$ \\
\hline & $\mathbf{B}_{1}$ & 2.5 & 2.5 & Hold and Maintain (HM) & $\{\mathrm{HD}(0), \mathrm{HM}(1), \mathrm{GB}(0)\}$ \\
\hline & $\mathbf{B}_{2}$ & 2.8 & 2.8 & Hold and Maintain (HM) & $\begin{array}{l}\{\mathrm{HD}(0), \mathrm{HM}(0.525), \mathrm{GB} \\
(0.475)\}\end{array}$ \\
\hline \multirow{2}{*}{ III } & $\mathrm{C}_{1}$ & 2.0 & 3.0 & Hold and Maintain (HM) & $\{\mathrm{HD}(0), \mathrm{HM}(1), \mathrm{GB}(0)\}$ \\
\hline & $\mathbf{C}_{2}$ & 2.2 & 2.8 & Hold and Maintain (HM) & $\{\mathrm{HD}(0), \mathrm{HM}(1), \mathrm{GB}(0)\}$ \\
\hline \multirow[b]{2}{*}{ IV } & $\mathrm{D}_{1}$ & 2.5 & 3.5 & Grow and Build (GB) & $\{\mathrm{HD}(0), \mathrm{HM}(0), \mathrm{GB}(1)\}$ \\
\hline & $\mathbf{D}_{2}$ & 2.2 & 3.8 & Hold and Maintain (HM) & $\begin{array}{l}\{\mathrm{HD}(0), \mathrm{HM}(0.72), \mathrm{GB} \\
(0.28)\}\end{array}$ \\
\hline
\end{tabular}

RESULTS OF NON-FUZZY AND FUZZY DECISION ON STRATEGY FEASIBILITY 
In the case of region III i.e. at intersections external to cells, $\mathrm{C}_{1}(I F A=2.0$ and $E F A=3.0)$ would treated as 'Hold and Maintain, $(\mathrm{HD}(0)$, $\mathrm{HM}(1)$ and $\mathrm{GB}(0)) ; \mathrm{C}_{2}$ would be treated as

'Hold and Maintain' as well (IFA=3.0, $E F A=2.0)(\mathrm{HD}(0), \mathrm{HM}(1), \mathrm{GB}(0))$.

Region IV whose ambiguity arouse to equivalent accumulated to the coordinate values as depicted as $D_{1}$ and $D_{2}$. This will be treated as follows: the case of $\mathrm{D}_{1}(I F A=2.5$, $E F A=3.5)$ will be treated as 'Grow and Build' $(\mathrm{HD}(0), \mathrm{HM}(0), \mathrm{GB}(1)$ whereas in the case of $\mathrm{D}_{2}(I F A=2.2, E F A=3.8)$, it will be treated as 'Hold and Maintain' $(\mathrm{HD}(0), \mathrm{HM}(0.72)$ and $\mathrm{GB}(0.28))$ due to the 'Hold and Maintain' value 0.72

In a difficult situation such $\mathrm{C}_{1}$ and $\mathrm{C}_{2}$, in which there is the similar strategic choice of 'Hold and Maintain', one would have to resort to the 'market' or 'resource-driven assumptions. If 'market-driven' then the choice would be $\mathrm{C}_{1}$ due to high $E F A$ values. However if the basic assumption is for resource-based driven, then $\mathrm{C}_{2}$ will be chosen due to its higher $I F A$ compared $E F A$ value.

\section{SFS : STRATEGIC FORMULATION SYSTEM}

For implementation purposes, a prototype of strategic analysis system namely, $S F S$ has been developed. The system requires user to enter the SWOT factors. In addition, the rank and the rate of each factor are also required to calculate both IFA and EFA scores (as Fig 12).

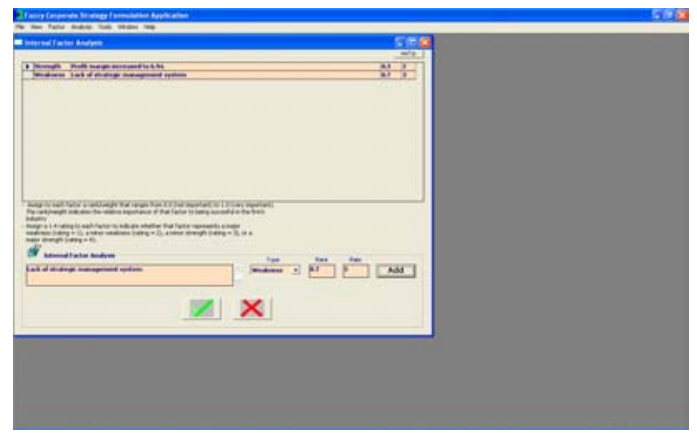

Fig. 12. IFA analysis interface

IFA and EFA score are deduced by calculating the total weighted value of internal and external factors respectively (as 3 and 4).

IFA score $=\sum$ internal $_{\mathrm{i}}$.rank $\bullet$ internal $_{\mathrm{i}}$.rate
$E F A$ score $=\sum$ external $_{\mathrm{i}} \cdot$ rank $\bullet$ external $_{\mathrm{i}} \cdot$ rate (4)

The total weighted score of IFA and EFA are then mapped onto the I-E matrix. In forecasting the appropriate strategy, both scores are fuzzified and the certainty value of implementing the appropriate strategy will be produced by the system. This provides extra information to a particular company in determining which strategy to undertake. If the implementation of chosen strategy shows to be impossible, a strategy with next highest degree of membership can be used. The interface of strategy mapping is depicted as Fig. 13.

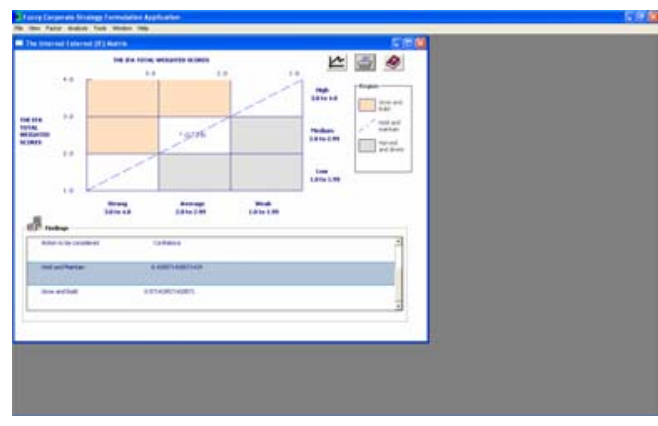

Fig. 13. Strategy mapping interface with confidence value

\section{SUMMARY AND CONCLUSIONS}

There are various ambiguous regions in an IEMatrix. This study seems to show that Fuzzy Logic is an appropriate technique to improve decision-making in locating the overall corporate strategy for an organization. The values describing business position of a company have been fuzzified and the analysis of this position has been carried out. Results obtained in this way are more useful than the ones offered by the classical approach. It can accurate the strategy location for a company whether the company should Grow and Build, Hold and Maintain or Harvest and Divest. It is also able to forecast the strategic choice for the ambiguous locations that exists in the company given a 'market driven' or 'resourcebased' assumption. Given the deduced certainty factor, Fuzzy Logic can also provide the predicted value of location, thus providing extra information for the strategic decisionmaker to make his next move. Using Fuzzy Logic based decision support system results in not only possibility of faster counteraction to the changes on the market, but also in better overview of applicable strategies and prospects for their combining. The ability to map the present situation of the company and 
to predict possible future developments could be useful for companies based their strategy on the analysis. The stakeholders of the company are dependent of the quality with which a company estimates its future and the strategies that are based on these expectations.

\section{REFERENCES}

[1] Azizi Ab. Aziz, Azizi Zakaria and Fadzilah Siraj. (2002). Improving and Supporting Decision Making Using Intelligent Techniques. Proceedings of The Third Annual Seminar Dynamics of Change, Universiti Utara Malaysia.

[2] David, F. (1999). Strategic Management. Addison Wesley, Boston, USA.

[3] Harris, J. (2001). An Introduction to Fuzzy Logic Applications, Kluwer Academic Publishers, ISBN 1-4020-0395-1.

[4] Jang, J. -S., Sun, C. -T. and Mizutani, E. (1997). Neuro-Fuzzy and Soft Computing : A Computational Approach to Learning and Machine Intelligence, MATLAB Curriculum Series, Prentice Hall.

[5] Lather, A.S., Kumar, S. and Singh, Y. (2000). Suitability Assessment of Software Developers: A fuzzy Approach. Software Engineering, 25(3), ACM SIGSOFT.
[6] Siti Maimon Kamso. (2003). Managing Corporate Strategies : From SWOT to SWOTIT. Universiti Utara Malaysia, Kedah, Malaysia.

[7] Siti Maimon Kamso and Chee Wei Ming. (2002). Applying the SWOT-TOWS Paradigm to Business (B2B) Collaboration : Relevence to Small and Medium Enterprises (SME). Juhary Ali, Wan Rafaei Abdul Rahman and Abdul Aziz Latif, (Eds.). Proceeding of the $6^{\text {th }}$ Annual Asia-Pacific Forum for Small Business, Oct, 16-18 at Corus Hotel, Kuala Lumpur.

[8] Tsoukalas, L.H. and Utirig, E. (1997). Fuzzy and Neural Approaches in Engineering, John \& Wiley.

[9] Wheelen, T.L. and Hunger, J.D. (1998), Strategic Management and Business Policy $\quad\left(6^{\text {th }} . \quad E d\right)$. Addison-Wesley Publishing Company, Inc.

[10] Yager, R. and Lofti A. Zadeh. (1992). An Introduction to Fuzzy Logic Applications in Intelligent System, Kluwer Academic Publishers. 